\title{
ON GENERALIZED JORDAN PREDERIVATIONS AND GENERALIZED PREDERIVATIONS OF LIE COLOR ALGEBRAS
}

\author{
CHENRUI YAO, YAO MA, AND LIANGYUN CHEN
}

\begin{abstract}
In this paper, the concepts of (generalized) $(\theta, \varphi)$-prederivations and (generalized) Jordan $(\theta, \varphi)$-prederivations on a Lie color algebra are introduced. It is proved that Jordan $(\theta, \varphi)$-prederivations (resp. generalized Jordan $(\theta, \varphi)$-prederivations) are $(\theta, \varphi)$-prederivations (resp. generalized $(\theta, \varphi)$ prederivations) on a Lie color algebra under some conditions. In particular, Jordan $\theta$-prederivations are $\theta$-prederivations on a Lie color algebra.
\end{abstract}

\section{INTRODUCTION}

Lie algebras and Lie superalgebras play important roles in many mathematical and physical subjects, e.g. differential geometry, Yang-Baxter equations, etc. A well known use of the generalizations of Lie theory corresponds to the study of symmetries into physics. As the natural generalization of Lie algebras and Lie superalgebras, Rittenberg and Wyler [20] introduced the notion of Lie color algebras in 1978, and discussed their possible physical applications. In 1979, Scheunert [21] investigated the Lie color algebras from a purely mathematical point of view and obtained generalizations of the PBW and Ado theorems. Montgomery [16] obtained the simple Lie color algebras from associative graded algebras and proved Herstein's theorem. In recent years, Lie color algebras have become an interesting subject of mathematics and physics [2, 8, 22, 24].

Let $L$ be a Lie algebra. A derivation of $L$ is a linear mapping $D \in \operatorname{Hom}(L, L)$ satisfying

$$
D([x y])=[D(x) y]+[x D(y)] .
$$

Further, the notion of derivation was generalized in various directions, e.g., Leger and Luks 12 considered the notion of generalized derivation, that is, $D \in \operatorname{Hom}(L, L)$ such that there exist $D^{\prime}, D^{\prime \prime} \in \operatorname{Hom}(L, L)$ with

$$
[D(x) y]+\left[x D^{\prime}(y)\right]=D^{\prime \prime}([x y]) .
$$

2010 Mathematics Subject Classification. 17B40, 17A40, 17B75.

Key words and phrases. Derivations, Prederivations, Lie color algebras.

Supported by NNSF of China (Nos. 11471090, 11771069, 11401425), NSF of Jilin Province (No. 20170101048JC), the project of Jilin Province Department of Education (No. JJKH20180005K) and the Fundamental Research Funds for the Central Universities (No. 130014801). 
Derivations and generalized derivations can be used to characterize algebras, so there has been a great deal of work concerning them. For instance, Jacobson [10] proved that a Lie algebra of characteristic zero with a nonsingular derivation must be nilpotent; they also play an important role in the existence question of left-invariant affine structures on Lie groups, etc.

On the other hand, there are various generalizations of derivations, in which prederivations (or Lie triple derivations) have received a fair amount of attention. Note that a prederivation of a Lie algebra $L$ is a linear mapping $D: L \rightarrow L$ such that

$$
D([[x, y], z])=[[D(x), y], z]+[[x, D(y)], z]+[[x, y], D(z)], \quad \forall x, y, z \in L .
$$

Müller [17] introduced the concept of prederivations (or Lie triple derivations) of Lie algebras, and he showed that if $G$ is a Lie group endowed with a bi-invariant semi-Riemannian metric and $g$ its Lie algebra, then the Lie algebra of the group of isometries of $G$ satisfying the identity element is a subalgebra of the prederivation algebra of $g$. Bajo [1] showed that a real Lie algebra with a non-singular prederivation is necessarily nilpotent, and this was also true for complex Lie algebras. Moens [15] generalized these results to Leibniz derivations over any field of characteristic zero. For additional essential contributions to the related topics, refer to [11, 14, 4, 5, 6, 23, 25.

Another interesting topic related to generalized derivations is under which conditions Jordan generalized derivation becomes a generalized derivation. Notice that if $R$ is an associative ring, then an additive mapping $D: R \rightarrow R$ is called a Jordan derivation if

$$
D\left(a^{2}\right)=D(a) a+a D(a), \quad \forall a \in R .
$$

Similarly, a Jordan prederivation (or Jordan triple derivation) of a Lie algebra $L$ is a linear mapping $D: L \rightarrow L$ such that

$$
D([[x, y], x])=[[D(x), y], x]+[[x, D(y)], x]+[[x, y], D(x)], \quad \forall x, y \in L .
$$

Obviously, every derivation (resp. prederivation) is a Jordan derivation (resp. Jordan prederivation). The converse is not true in general. Herstein [9] proved that every Jordan derivation on a 2-torsion free prime ring is a derivation. Brešar [3] extended this result to 2-torsion free semiprime rings. Inspired by [13, 18, 19], in this paper we'll investigate under which conditions Jordan $(\theta, \varphi)$-prederivations (resp. generalized Jordan $(\theta, \varphi)$-prederivations) are $(\theta, \varphi)$-prederivations (resp. generalized $(\theta, \varphi)$-prederivations) on a Lie color algebra.

First, we recall some elementary definitions.

Definition 1.1 (See [7]). Let $F$ be a field and $G$ an abelian group. A mapping $\varepsilon$ : $G \times G \rightarrow F^{*}$ is called a skew-symmetric bicharacter on $G$ if the following identities hold, for all $f, g, h \in G$ :

(i) $\varepsilon(f, g+h)=\varepsilon(f, g) \varepsilon(f, h)$,

(ii) $\varepsilon(g+h, f)=\varepsilon(g, f) \varepsilon(h, f)$,

(iii) $\varepsilon(g, h) \varepsilon(h, g)=1$. 
Definition 1.2 (See [7). A Lie color algebra $L$ is a $G$-graded vector space $L=$ $\bigoplus_{g \in G} L_{g}$ with a graded bilinear mapping [, ] : $L \times L \rightarrow L$ satisfying

(i) $\left[L_{\theta}, L_{\mu}\right] \subseteq L_{\theta+\mu}, \forall \theta, \mu \in G$,

(ii) $[x, y]=-\varepsilon(x, y)[y, x]$,

(iii) $\varepsilon(z, x)[x,[y, z]]+\varepsilon(x, y)[y,[z, x]]+\varepsilon(y, z)[z,[x, y]]=0$,

where $x \in L_{\sigma(x)}, y \in L_{\sigma(y)}, z \in L_{\sigma(z)}, \sigma(x), \sigma(y), \sigma(z) \in G$.

Example 1.3. Let $G$ be the group $Z_{2} \times Z_{2} \times Z_{2}$. For convenience, we set $e_{1}=$ $(1,1,0), e_{2}=(1,0,1), e_{3}=(0,1,1)$. Let $L=F e_{1} \dot{+} F e_{2} \dot{+} F e_{3}$ and let the multiplication table of the basic elements be

$$
\left[e_{1}, e_{2}\right]=e_{3},\left[e_{1}, e_{3}\right]=e_{2},\left[e_{2}, e_{3}\right]=\left[e_{1}, e_{1}\right]=\left[e_{2}, e_{2}\right]=\left[e_{3}, e_{3}\right]=0 .
$$

We define $\varepsilon: G \times G \mapsto F^{*}$ by the matrix

$$
[\varepsilon(i, j)]=\left[\begin{array}{ccc}
1 & -1 & -1 \\
-1 & 1 & -1 \\
-1 & -1 & 1
\end{array}\right],
$$

where elements of this matrix specify in the natural way values of $\varepsilon$ on the set $\{(1,1,0),(1,0,1),(0,1,1)\} \times\{(1,1,0),(1,0,1),(0,1,1)\} \subset G \times G$. (The elements $(1,1,0),(1,0,1),(0,1,1)$ are ordered and numbered by the numbers $1,2,3$ respectively.) The values of $\varepsilon$ on other elements from $G$ do not affect the multiplication above. It is easily seen that the function $\varepsilon$ can be extended to the whole $G \times G$ so that $L$ is a Lie color algebra.

Remark 1.4. Our main result Theorem 2.4 needs the condition that

$$
\varepsilon(x, z)+\varepsilon(y, x)+\varepsilon(z, y) \varepsilon(y, x) \neq 0, \quad \forall x, y, z \in L .
$$

So throughout this paper, the Lie color algebra $L$ is assumed to satisfy the above equality. Hence we should show that such Lie color algebras do exist. However, we could immediately see that the Lie color algebra in Example 1.3 works, which implies that our condition is reasonable.

\section{MAIN RESUlts}

Definition 2.1. Let $L$ be a Lie color algebra and let $D, \theta, \varphi: L \rightarrow L$ be homogeneous linear mappings, where $d(\theta)=d(\varphi)=0$. We denote by $d(D)$ the $G$-graded degree of a homogeneous linear mapping $D$ of $L$.

(1) $D$ is called a $(\theta, \varphi)_{1}$-prederivation if

$$
\begin{aligned}
D([[x, y], z])= & {[[D(x), \theta(y)], \varphi(z)]+\varepsilon(D, x)[[\theta(x), D(y)], \varphi(z)] } \\
& +\varepsilon(D, x) \varepsilon(D, y)[[\theta(x), \varphi(y)], D(z)], \quad \forall x, y, z \in L .
\end{aligned}
$$

(2) $D$ is called a $(\theta, \varphi)_{2}$-prederivation if

$$
\begin{aligned}
D([[x, y], z])= & {[[D(x), \theta(y)], \theta(z)]+\varepsilon(D, x)[[\theta(x), D(y)], \varphi(z)] } \\
& +\varepsilon(D, x) \varepsilon(D, y)[[\varphi(x), \varphi(y)], D(z)], \quad \forall x, y, z \in L .
\end{aligned}
$$


(3) $D$ is called a $(\theta, \varphi)_{3}$-prederivation if

$$
\begin{aligned}
D([[x, y], z])= & {[[D(x), \theta(y)], \theta(z)]+\varepsilon(D, x)[[\varphi(x), D(y)], \theta(z)] } \\
& +\varepsilon(D, x) \varepsilon(D, y)[[\varphi(x), \varphi(y)], D(z)], \quad \forall x, y, z \in L .
\end{aligned}
$$

In particular, $\forall i=1,2,3$, a $(\theta, \varphi)_{i}$-prederivation $D$ is called a $\theta$-prederivation if $\theta=\varphi$. It is clear that a $(\theta, \varphi)_{i}$-prederivation is a prederivation when $\theta=\varphi=1_{T}$.

Example 2.2. We take the Lie color algebra in Example 1.3 Let $D$ be a homogeneous linear mapping on $L$, satisfying $D\left(e_{1}\right)=0, D\left(e_{2}\right)=\lambda e_{3}, D\left(e_{3}\right)=\mu e_{2}$. Obviously, while $\lambda \neq-\mu, D$ is not a derivation on $L$, since

$$
\left[D\left(e_{1}\right), e_{2}\right]+\varepsilon\left(e_{1}, e_{2}\right)\left[e_{1}, D\left(e_{2}\right)\right]=-\lambda e_{2} \neq \mu e_{2}=D\left(e_{3}\right)=D\left(\left[e_{1}, e_{2}\right]\right) .
$$

But one can show that $D$ is a prederivation on $L$, so there exist nontrivial (i.e. non-derivation) prederivations on a Lie color algebra.

Moreover, let $D, \theta, \varphi$ be a homogeneous linear mapping on $L$ satisfying

$$
\begin{gathered}
D\left(e_{1}\right)=0, \quad D\left(e_{2}\right)=\lambda e_{3}, \quad D\left(e_{3}\right)=\mu e_{2} \\
\theta\left(e_{1}\right)=a e_{1}, \quad \theta\left(e_{2}\right)=b e_{2}, \quad \theta\left(e_{3}\right)=c e_{3} \\
\varphi\left(e_{1}\right)=\alpha e_{1}, \quad \varphi\left(e_{2}\right)=\beta e_{2}, \quad \varphi\left(e_{3}\right)=\gamma e_{3} .
\end{gathered}
$$

When $\lambda \neq-\mu$ and $a \alpha=1, D$ is a $(\theta, \varphi)_{1}$-prederivation on $L$. Similarly, one may construct $(\theta, \varphi)_{2}$-prederivations and $(\theta, \varphi)_{3}$-prederivations on $L$.

Definition 2.3. Let $L$ be a Lie color algebra and let $D, \theta, \varphi: L \rightarrow L$ be homogeneous linear mappings, where $d(\theta)=d(\varphi)=0$.

(1) $D$ is called a Jordan $(\theta, \varphi)_{1}$-prederivation if

$$
\begin{aligned}
D([[x, y], x])= & {[[D(x), \theta(y)], \varphi(x)]+\varepsilon(D, x)[[\theta(x), D(y)], \varphi(x)] } \\
& +\varepsilon(D, x) \varepsilon(D, y)[[\theta(x), \varphi(y)], D(x)], \quad \forall x, y \in L .
\end{aligned}
$$

(2) $D$ is called a Jordan $(\theta, \varphi)_{2}$-prederivation if

$$
\begin{aligned}
D([[x, y], x])= & {[[D(x), \theta(y)], \theta(x)]+\varepsilon(D, x)[[\theta(x), D(y)], \varphi(x)] } \\
& +\varepsilon(D, x) \varepsilon(D, y)[[\varphi(x), \varphi(y)], D(x)], \quad \forall x, y \in L .
\end{aligned}
$$

(3) $D$ is called a Jordan $(\theta, \varphi)_{3}$-prederivation if

$$
\begin{aligned}
D([[x, y], x])= & {[[D(x), \theta(y)], \theta(x)]+\varepsilon(D, x)[[\varphi(x), D(y)], \theta(x)] } \\
& +\varepsilon(D, x) \varepsilon(D, y)[[\varphi(x), \varphi(y)], D(x)], \quad \forall x, y \in L .
\end{aligned}
$$

In particular, $\forall i=1,2,3$, a Jordan $(\theta, \varphi)_{i}$-prederivation $D$ is called a Jordan $\theta$ prederivation if $\theta=\varphi$. It is clear that a Jordan $(\theta, \varphi)_{i}$-prederivation is a Jordan prederivation when $\theta=\varphi=1_{T}$.

It is clear that if $D_{i}$ is a $(\theta, \varphi)_{i}$-prederivation of $L$, then $D_{i}$ is a Jordan $(\theta, \varphi)_{i^{-}}$ prederivation of $L$, where $i=1,2,3$.

In this section, $L$ is a Lie color algebra and $\theta, \varphi$ are defined to be homogeneous linear mappings of $L$ satisfying $d(\theta)=d(\varphi)=0$.

Theorem 2.4. $D$ is a $(\theta, \varphi)_{1}$-prederivation of $L$ if and only if $D$ is a Jordan $(\theta, \varphi)_{1}$-prederivation of $L$ such that 
(i) $[[\theta(x), \varphi(y)], D(z)]=[[\varphi(x), \theta(y)], D(z)]$,

(ii) $\varepsilon(z, x) A(x, y, z)+\varepsilon(x, y) A(y, z, x)+\varepsilon(y, z) A(z, x, y)=0$,

where $x, y, z \in L$ and $A(x, y, z)=[[D(x), \theta(y)], \varphi(z)]+\varepsilon(D, x)[[\theta(x), D(y)], \varphi(z)]+$ $\varepsilon(D, x) \varepsilon(D, y)[[\theta(x), \varphi(y)], D(z)]$.

Proof. Assume that $D$ is a $(\theta, \varphi)_{1}$-prederivation of $L$. Clearly, $D$ is a Jordan $(\theta, \varphi)_{1}$-prederivation of $L$ and $D([[x, y], z])=-\varepsilon(x, y) D([[y, x], z])$; note that

$$
\begin{aligned}
-\varepsilon(x, y) D([[y, x], z])= & -\varepsilon(x, y)[[D(y), \theta(x)], \varphi(z)] \\
& -\varepsilon(x, y) \varepsilon(D, y)[[\theta(y), D(x)], \varphi(z)] \\
& -\varepsilon(x, y) \varepsilon(D, x) \varepsilon(D, y)[[\theta(y), \varphi(x)], D(z)] \\
= & {[[D(x), \theta(y)], \varphi(z)]+\varepsilon(D, x)[[\theta(x), D(y)], \varphi(z)] } \\
& +\varepsilon(D, x) \varepsilon(D, y)[[\varphi(x), \theta(y)], D(z)]
\end{aligned}
$$

and

$$
\begin{aligned}
D([[x, y], z])= & {[[D(x), \theta(y)], \varphi(z)]+\varepsilon(D, x)[[\theta(x), D(y)], \varphi(z)] } \\
& +\varepsilon(D, x) \varepsilon(D, y)[[\theta(x), \varphi(y)], D(z)]
\end{aligned}
$$

then (i) follows. Since $D$ is a $(\theta, \varphi)_{1}$-prederivation of $L$, we have $D([[x, y], z])=$ $A(x, y, z)$; hence

$$
\begin{aligned}
\varepsilon(z, x) & A(x, y, z)+\varepsilon(x, y) A(y, z, x)+\varepsilon(y, z) A(z, x, y) \\
& =\varepsilon(z, x) D([[x, y], z])+\varepsilon(x, y) D([[y, z], x])+\varepsilon(y, z) D([[z, x], y]) \\
& =D(\varepsilon(z, x)[[x, y], z]+\varepsilon(x, y)[[y, z], x]+\varepsilon(y, z)[[z, x], y]) \\
& =0 .
\end{aligned}
$$

Conversely, let $D$ be a Jordan $(\theta, \varphi)_{1}$-prederivation of $L$ for which (i) and (ii) hold. Then $D([[x, y], x])=A(x, y, x)$. It follows that

$$
\begin{aligned}
D([[x+z, y], x+z]) & =D([[x, y], x])+D([[x, y], z])+D([[z, y], x])+D([[z, y], z]) \\
& =A(x, y, x)+A(z, y, z)+D([[x, y], z])+D([[z, y], x])
\end{aligned}
$$

and

$$
\begin{aligned}
D([[x+z, y], x+z]) & =A(x+z, y, x+z) \\
& =A(x, y, x)+A(x, y, z)+A(z, y, x)+A(z, y, z) .
\end{aligned}
$$

Thus we obtain

$$
D([[x, y], z])+D([[z, y], x])=A(x, y, z)+A(z, y, x)
$$


By (i),

$$
\begin{aligned}
A(y, x, z)= & {[[D(y), \theta(x)], \varphi(z)]+\varepsilon(D, y)[[\theta(y), D(x)], \varphi(z)] } \\
& +\varepsilon(D, x) \varepsilon(D, y)[[\theta(y), \varphi(x)], D(z)] \\
= & -\varepsilon(y, x)[[D(x), \theta(y)], \varphi(z)]-\varepsilon(y, x) \varepsilon(D, x)[[\theta(x), D(y)], \varphi(z)] \\
& -\varepsilon(y, x) \varepsilon(D, x) \varepsilon(D, y)[[\varphi(x), \theta(y)], D(z)] \\
= & -\varepsilon(y, x)[[D(x), \theta(y)], \varphi(z)]-\varepsilon(y, x) \varepsilon(D, x)[[\theta(x), D(y)], \varphi(z)] \\
& -\varepsilon(y, x) \varepsilon(D, x) \varepsilon(D, y)[[\theta(x), \varphi(y)], D(z)] \\
= & -\varepsilon(y, x) A(x, y, z) .
\end{aligned}
$$

This implies that

$$
D([[x, y], y])=-\varepsilon(x, y) D([[y, x], y])=-\varepsilon(x, y) A(y, x, y)=A(x, y, y) .
$$

A similar argument proves

$$
D([[x, y], z])+D([[x, z], y])=A(x, y, z)+A(x, z, y) .
$$

By $* * \varepsilon(x, z)+* * \times \varepsilon(y, x)$, we have

$$
\begin{array}{r}
\varepsilon(x, z)(D([[x, y], z])+D([[z, y], x]))+\varepsilon(y, x)(D([[x, y], z])+D([[x, z], y])) \\
=\varepsilon(x, z)(A(x, y, z)+A(z, y, x))+\varepsilon(y, x)(A(x, y, z)+A(x, z, y)),
\end{array}
$$

then

$$
\begin{aligned}
\varepsilon(x, z) D([[x, y], z])+\varepsilon(y, x) D([[x, y], z])-\varepsilon(z, y) D([[y, x], z]) & \\
= & \varepsilon(x, z)(A(x, y, z)+A(z, y, x))+\varepsilon(y, x)(A(x, y, z)+A(x, z, y)),
\end{aligned}
$$

that is,

$$
\begin{aligned}
(\varepsilon(x, z)+\varepsilon & (y, x)+\varepsilon(z, y) \varepsilon(y, x)) D([[x, y], z]) \\
= & (\varepsilon(x, z)+\varepsilon(y, x)+\varepsilon(z, y) \varepsilon(y, x)) A(x, y, z) \\
& +\varepsilon(x, z) A(z, y, x)+\varepsilon(y, x) A(x, z, y)-\varepsilon(z, y) \varepsilon(y, x) A(x, y, z) \\
= & (\varepsilon(x, z)+\varepsilon(y, x)+\varepsilon(z, y) \varepsilon(y, x)) A(x, y, z) \\
& +\varepsilon(x, z) A(z, y, x)+\varepsilon(y, x) A(x, z, y)+\varepsilon(z, y) A(y, x, z) \\
= & (\varepsilon(x, z)+\varepsilon(y, x)+\varepsilon(z, y) \varepsilon(y, x)) A(x, y, z),
\end{aligned}
$$

where the last equality uses (ii). Since $\varepsilon(x, z)+\varepsilon(y, x)+\varepsilon(z, y) \varepsilon(y, x) \neq 0$, we have $D([[x, y], z])=A(x, y, z)$, i.e., $D$ is a $(\theta, \varphi)_{1}$-prederivation of $L$.

Corollary 2.5. $D$ is a $\theta$-prederivation of $L$ if and only if $D$ is a Jordan $\theta$ prederivation of $L$.

Proof. If $D$ is a Jordan $\theta$-prederivation of $L$, then (i) follows immediately. (ii) holds because

$$
\begin{aligned}
& \varepsilon(z, x) A(x, y, z)+\varepsilon(x, y) A(y, z, x)+\varepsilon(y, z) A(z, x, y) \\
& =\varepsilon(z, x)[[D(x), \theta(y)], \theta(z)]+\varepsilon(z, x) \varepsilon(D, x)[[\theta(x), D(y)], \theta(z)] \\
& \quad+\varepsilon(z, x) \varepsilon(D, x) \varepsilon(D, y)[[\theta(x), \theta(y)], D(z)]
\end{aligned}
$$




$$
\begin{aligned}
+ & \varepsilon(x, y)[[D(y), \theta(z)], \theta(x)]+\varepsilon(x, y) \varepsilon(D, y)[[\theta(y), D(z)], \theta(x)] \\
+ & \varepsilon(x, y) \varepsilon(D, y) \varepsilon(D, z)[[\theta(y), \theta(z)], D(x)] \\
+ & \varepsilon(y, z)[[D(z), \theta(x)], \theta(y)]+\varepsilon(y, z) \varepsilon(D, z)[[\theta(z), D(x)], \theta(y)] \\
+ & \varepsilon(y, z) \varepsilon(D, x) \varepsilon(D, z)[[\theta(z), \theta(x)], D(y)] \\
= & \varepsilon(D, z)(\varepsilon(z, D) \varepsilon(z, x)[[D(x), \theta(y)], \theta(z)]+\varepsilon(x, y) \varepsilon(D, y)[[\theta(y), \theta(z)], D(x)] \\
& +\varepsilon(y, z)[[\theta(z), D(x)], \theta(y)]) \\
+ & \varepsilon(D, x)(\varepsilon(x, D) \varepsilon(x, y)[[D(y), \theta(z)], \theta(x)]+\varepsilon(y, z) \varepsilon(D, z)[[\theta(z), \theta(x)], D(y)] \\
& +\varepsilon(z, x)[[\theta(x), D(y)], \theta(z)]) \\
+ & \varepsilon(D, y)(\varepsilon(y, D) \varepsilon(y, z)[[D(z), \theta(x), \theta(y)]]+\varepsilon(z, x) \varepsilon(D, x)[[\theta(x), \theta(y)], D(z)] \\
& \quad+\varepsilon(x, y)[[\theta(y), D(z)], \theta(x)]) \\
= & 0 .
\end{aligned}
$$

Therefore, $D$ is a $\theta$-prederivation of $L$ by Theorem 2.4

Theorem 2.6. $D$ is a $(\theta, \varphi)_{2}$-prederivation of $L$ if and only if $D$ is a Jordan $(\theta, \varphi)_{2}$-prederivation of $L$ such that

(i) $[[D(x), \theta(y)],(\varphi-\theta)(z)]=\varepsilon(D, x)[[\theta(x), D(y)],(\varphi-\theta)(z)]$,

(ii) $\varepsilon(z, x) A^{\prime}(x, y, z)+\varepsilon(x, y) A^{\prime}(y, z, x)+\varepsilon(y, z) A^{\prime}(z, x, y)=0$,

where $x, y, z \in L$ and $A^{\prime}(x, y, z)=[[D(x), \theta(y)], \theta(z)]+\varepsilon(D, x)[[\theta(x), D(y)], \varphi(z)]+$ $\varepsilon(D, x) \varepsilon(D, y)[[\varphi(x), \varphi(y)], D(z)]$.

Proof. Let $D$ be a $(\theta, \varphi)_{2}$-prederivation of $L$. Use the fact that

$$
D([[x, y], z])=-\varepsilon(x, y) D([[y, x], z]),
$$

as well as the fact that

$$
\begin{aligned}
-\varepsilon(x, y) D([[y, x], z])= & -\varepsilon(x, y)[[D(y), \theta(x)], \theta(z)]-\varepsilon(x, y) \varepsilon(D, y)[[\theta(y), D(x)], \varphi(z)] \\
& -\varepsilon(x, y) \varepsilon(D, x) \varepsilon(D, y)[[\varphi(y), \varphi(x)], D(z)] \\
= & {[[D(x), \theta(y)], \varphi(z)]+\varepsilon(D, x)[[\theta(x), D(y)], \theta(z)] } \\
& +\varepsilon(D, x) \varepsilon(D, y)[[\varphi(x), \varphi(y)], D(z)],
\end{aligned}
$$

then we have

$$
\begin{aligned}
{[[D(x), \theta(y)], \theta(z)]+\varepsilon(D, x) } & {[[\theta(x), D(y)], \varphi(z)] } \\
& =[[D(x), \theta(y)], \varphi(z)]+\varepsilon(D, x)[[\theta(x), D(y)], \theta(z)],
\end{aligned}
$$

that is, $[[D(x), \theta(y)],(\varphi-\theta)(z)]=\varepsilon(D, x)[[\theta(x), D(y)],(\varphi-\theta)(z)]$. It is routine to prove (ii).

Suppose, conversely, that $D$ is a Jordan $(\theta, \varphi)_{2}$-prederivation of $L$ satisfying (i) and (ii). Note that

$$
\begin{aligned}
A^{\prime}(y, x, z)= & {[[D(y), \theta(x)], \theta(z)]+\varepsilon(D, y)[[\theta(y), D(x)], \varphi(z)] } \\
& +\varepsilon(D, x) \varepsilon(D, y)[[\varphi(y), \varphi(x)], D(z)] \\
= & -\varepsilon(y, x)[[D(x), \theta(y)], \varphi(z)]-\varepsilon(y, x) \varepsilon(D, x)[[\theta(x), D(y)], \theta(z)]
\end{aligned}
$$




$$
\begin{aligned}
& -\varepsilon(y, x) \varepsilon(D, x) \varepsilon(D, y)[[\varphi(x), \varphi(y)], D(z)] \\
= & -\varepsilon(y, x)[[D(x), \theta(y)], \theta(z)]-\varepsilon(y, x) \varepsilon(D, x)[[\theta(x), D(y)], \varphi(z)] \\
& -\varepsilon(y, x) \varepsilon(D, x) \varepsilon(D, y)[[\varphi(x), \varphi(y)], D(z)] \\
= & -\varepsilon(y, x) A^{\prime}(x, y, z) .
\end{aligned}
$$

In the same way, we can get equalities $(*)$ and (**). The rest of the proof is the same as the corresponding proof of Theorem 2.4 .

A similar argument proves the following result.

Theorem 2.7. $D$ is a $(\theta, \varphi)_{3}$-prederivation of $L$ if and only if $D$ is a Jordan $(\theta, \varphi)_{3}$-prederivation of $L$ such that

(i) $[[D(x),(\theta-\varphi)(y)], \theta(z)]=\varepsilon(D, x)[[(\theta-\varphi)(x), D(y)], \theta(z)]$,

(ii) $\varepsilon(z, x) A^{\prime \prime}(x, y, z)+\varepsilon(x, y) A^{\prime \prime}(y, z, x)+\varepsilon(y, z) A^{\prime \prime}(z, x, y)=0$,

where $x, y, z \in L$ and $A^{\prime \prime}(x, y, z)=[[D(x), \theta(y)], \theta(z)]+\varepsilon(D, x)[[\varphi(x), D(y)], \theta(z)]+$ $\varepsilon(D, x) \varepsilon(D, y)[[\varphi(x), \varphi(y)], D(z)]$.

Remark 2.8. Corollary 2.5 can also be concluded from Theorem 2.6 or Theorem 2.7 since for any $x, y, z \in L, A(x, y, z)=A^{\prime}(x, y, z)=A^{\prime \prime}(x, y, z)$ when $D$ is a Jordan $\theta$-prederivation.

Definition 2.9. Let $L$ be a Lie color algebra.

(1) A generalized $(\theta, \varphi)_{1}$-prederivation with respect to a $(\theta, \varphi)_{1}$-prederivation $\delta_{1}$ is a homogeneous linear mapping $D_{1}: L \rightarrow L$ such that $d\left(D_{1}\right)=d\left(\delta_{1}\right)$ and

$$
\begin{aligned}
D_{1}([[x, y], z])= & {\left[\left[\delta_{1}(x), \theta(y)\right], \varphi(z)\right]+\varepsilon\left(D_{1}, x\right)\left[\left[\theta(x), \delta_{1}(y)\right], \varphi(z)\right] } \\
& +\varepsilon\left(D_{1}, x\right) \varepsilon\left(D_{1}, y\right)\left[[\theta(x), \varphi(y)], D_{1}(z)\right], \quad \forall x, y, z \in L .
\end{aligned}
$$

(2) A generalized $(\theta, \varphi)_{2}$-prederivation with respect to a $(\theta, \varphi)_{2}$-prederivation $\delta_{2}$ is a homogeneous linear mapping $D_{2}: L \rightarrow L$ such that $d\left(D_{2}\right)=d\left(\delta_{2}\right)$ and

$$
\begin{aligned}
D_{2}([[x, y], z])= & {\left[\left[\delta_{2}(x), \theta(y)\right], \theta(z)\right]+\varepsilon\left(D_{2}, x\right)\left[\left[\theta(x), \delta_{2}(y)\right], \varphi(z)\right] } \\
& +\varepsilon\left(D_{2}, x\right) \varepsilon\left(D_{2}, y\right)\left[[\varphi(x), \varphi(y)], D_{2}(z)\right], \quad \forall x, y, z \in L .
\end{aligned}
$$

(3) A generalized $(\theta, \varphi)_{3}$-prederivation with respect to a $(\theta, \varphi)_{3}$-prederivation $\delta_{3}$ is a homogeneous linear mapping $D_{3}: L \rightarrow L$ such that $d\left(D_{3}\right)=d\left(\delta_{3}\right)$ and

$$
\begin{aligned}
D_{3}([[x, y], z])= & {\left[\left[\delta_{3}(x), \theta(y)\right], \theta(z)\right]+\varepsilon\left(D_{3}, x\right)\left[\left[\varphi(x), \delta_{3}(y)\right], \theta(z)\right] } \\
& +\varepsilon\left(D_{3}, x\right) \varepsilon\left(D_{3}, y\right)\left[[\varphi(x), \varphi(y)], D_{3}(z)\right], \quad \forall x, y, z \in L .
\end{aligned}
$$

In particular, $\forall i=1,2,3$, a generalized $(\theta, \varphi)_{i}$-prederivation $D$ is called a generalized $\theta$-prederivation with respect to a $\theta$-prederivation $\delta$ if $\theta=\varphi$. It is clear that $D$ is a generalized prederivation when $\theta=\varphi=1_{T}$ and $\delta$ is a prederivation. 
Definition 2.10. Let $L$ be a Lie color algebra.

(1) A generalized Jordan $(\theta, \varphi)_{1}$-prederivation with respect to a Jordan $(\theta, \varphi)_{1}$ prederivation $\delta_{1}$ is a homogeneous linear mapping $D_{1}: L \rightarrow L$ such that $d\left(D_{1}\right)=d\left(\delta_{1}\right)$ and

$$
\begin{aligned}
D_{1}([[x, y], x])= & {\left[\left[\delta_{1}(x), \theta(y)\right], \varphi(x)\right]+\varepsilon\left(D_{1}, x\right)\left[\left[\theta(x), \delta_{1}(y)\right], \varphi(x)\right] } \\
& +\varepsilon\left(D_{1}, x\right) \varepsilon\left(D_{1}, y\right)\left[[\theta(x), \varphi(y)], D_{1}(x)\right], \quad \forall x, y \in L .
\end{aligned}
$$

(2) A generalized Jordan $(\theta, \varphi)_{2}$-prederivation with respect to a Jordan $(\theta, \varphi)_{2}$ prederivation $\delta_{2}$ is a homogeneous linear mapping $D_{2}: L \rightarrow L$ such that $d\left(D_{2}\right)=d\left(\delta_{2}\right)$ and

$$
\begin{aligned}
D_{2}([[x, y], x])= & {\left[\left[\delta_{2}(x), \theta(y)\right], \theta(x)\right]+\varepsilon\left(D_{2}, x\right)\left[\left[\theta(x), \delta_{2}(y)\right], \varphi(x)\right] } \\
& +\varepsilon\left(D_{2}, x\right) \varepsilon\left(D_{2}, y\right)\left[[\varphi(x), \varphi(y)], D_{2}(x)\right], \quad \forall x, y \in L .
\end{aligned}
$$

(3) A generalized Jordan $(\theta, \varphi)_{3}$-prederivation with respect to a Jordan $(\theta, \varphi)_{3}$ prederivation $\delta_{3}$ is a homogeneous linear mapping $D_{3}: L \rightarrow L$ such that $d\left(D_{3}\right)=d\left(\delta_{3}\right)$ and

$$
\begin{aligned}
D_{3}([[x, y], x])= & {\left[\left[\delta_{3}(x), \theta(y)\right], \theta(x)\right]+\varepsilon\left(D_{3}, x\right)\left[\left[\varphi(x), \delta_{3}(y)\right], \theta(x)\right] } \\
& +\varepsilon\left(D_{3}, x\right) \varepsilon\left(D_{3}, y\right)\left[[\varphi(x), \varphi(y)], D_{3}(x)\right], \quad \forall x, y \in L .
\end{aligned}
$$

In particular, $\forall i=1,2,3$, a generalized Jordan $(\theta, \varphi)_{i}$-prederivation $D$ is called a generalized Jordan $\theta$-prederivation with respect to a Jordan $\theta$-prederivation $\delta$ if $\theta=\varphi$. It is clear that $D$ is a generalized Jordan prederivation when $\theta=\varphi=1_{T}$ and $\delta$ is a Jordan prederivation.

Theorem 2.11. $D$ is a generalized $(\theta, \varphi)_{1}$-prederivation of $L$ with respect to a $(\theta, \varphi)_{1}$-prederivation $\delta$ if and only if $D$ is a generalized Jordan $(\theta, \varphi)_{1}$-prederivation of $L$ with respect to a $(\theta, \varphi)_{1}$-prederivation $\delta$ such that

(i) $[[\theta(x), \varphi(y)], D(z)]=[[\varphi(x), \theta(y)], D(z)]$,

(ii) $\varepsilon(z, x) B(x, y, z)+\varepsilon(x, y) B(y, z, x)+\varepsilon(y, z) B(z, x, y)=0$,

where $x, y, z \in L$ and $B(x, y, z)=[[\delta(x), \theta(y)], \varphi(z)]+\varepsilon(D, x)[[\theta(x), \delta(y)], \varphi(z)]+$ $\varepsilon(D, x) \varepsilon(D, y)[[\theta(x), \varphi(y)], D(z)]$.

Proof. Suppose that $D$ is a generalized $(\theta, \varphi)_{1}$-prederivation of $L$ with respect to a $(\theta, \varphi)_{1}$-prederivation $\delta$. Clearly, $D$ is a generalized Jordan $(\theta, \varphi)_{1}$-prederivation of $L$ and $D([[x, y], z])=-\varepsilon(x, y) D([[y, x], z])$. (i) follows from the fact that

$$
\begin{aligned}
-\varepsilon(x, y) D([[y, x], z])= & -\varepsilon(x, y)[[\delta(y), \theta(x)], \varphi(z)]-\varepsilon(x, y) \varepsilon(D, y)[[\theta(y), \delta(x)], \varphi(z)] \\
& -\varepsilon(x, y) \varepsilon(D, x) \varepsilon(D, y)[[\theta(y), \varphi(x)], D(z)] \\
= & {[[\delta(x), \theta(y)], \varphi(z)]+\varepsilon(D, x)[[\theta(x), \delta(y)], \varphi(z)] } \\
& +\varepsilon(D, x) \varepsilon(D, y)[[\varphi(x), \theta(y)], D(z)] .
\end{aligned}
$$


Since $D$ is a generalized $(\theta, \varphi)_{1}$-prederivation of $L$ with respect to $\delta$, we have $D([[x, y], z])=B(x, y, z)$; hence

$$
\begin{aligned}
\varepsilon(z, x) & (B(x, y, z)+\varepsilon(x, y) B(y, z, x)+\varepsilon(y, z) B(z, x, y) \\
& =\varepsilon(z, x) D([[x, y], z])+\varepsilon(x, y) D([[y, z], x])+\varepsilon(y, z) D([[z, x], y]) \\
& =D(\varepsilon(z, x)[[x, y], z]+\varepsilon(x, y)[[y, z], x]+\varepsilon(y, z)[[z, x], y]) \\
& =0 .
\end{aligned}
$$

Conversely, if $D$ is a generalized Jordan $(\theta, \varphi)_{1}$-prederivation of $L$ with respect to a $(\theta, \varphi)_{1}$-prederivation $\delta$ satisfying (i) and (ii), then (refer to the proof of Theorem 2.4 it suffices to prove that $B(x, y, z)=-\varepsilon(x, y) B(y, x, z)$. In fact,

$$
\begin{aligned}
B(y, x, z)= & {[[\delta(y), \theta(x)], \varphi(z)]+\varepsilon(D, y)[[\theta(y), \delta(x)], \varphi(z)] } \\
& +\varepsilon(D, y) \varepsilon(D, x)[[\theta(y), \varphi(x)], D(z)] \\
= & -\varepsilon(y, x)[[\delta(x), \theta(y)], \varphi(z)]-\varepsilon(y, x) \varepsilon(D, x)[[\theta(x), \delta(y)], \varphi(z)] \\
& -\varepsilon(y, x) \varepsilon(D, x) \varepsilon(D, y)[[\varphi(x), \theta(y)], D(z)] \\
= & -\varepsilon(y, x)[[\delta(x), \theta(y)], \varphi(z)]-\varepsilon(y, x) \varepsilon(D, x)[[\theta(x), \delta(y)], \varphi(z)] \\
& -\varepsilon(y, x) \varepsilon(D, x) \varepsilon(D, y)[[\theta(x), \varphi(y)], D(z)] \\
= & -\varepsilon(y, x) B(x, y, z) .
\end{aligned}
$$

This completes the proof.

Corollary 2.12. $D$ is a generalized $\theta$-prederivation of $L$ with respect to a $\theta$ prederivation $\delta$ if and only if $D$ is a generalized Jordan $\theta$-prederivation of $L$ with respect to a Jordan $\theta$-prederivation $\delta$ such that

$$
\begin{aligned}
\varepsilon(z, D) \varepsilon(z, x)[[\theta(x), \theta(y)],(D-\delta)(z)] & +\varepsilon(x, D) \varepsilon(x, y)[[\theta(y), \theta(z)],(D-\delta)(x)] \\
& +\varepsilon(y, D) \varepsilon(y, z)[[\theta(z), \theta(x)],(D-\delta)(y)]=0 .
\end{aligned}
$$

Proof. If $D$ is a generalized Jordan $\theta$-prederivation of $L$, then (i) follows immediately. (ii) holds because

$$
\begin{aligned}
\varepsilon(z, x) B( & x, y, z)+\varepsilon(x, y) B(y, z, x)+\varepsilon(y, z) B(z, x, y) \\
= & \varepsilon(z, x)[[\delta(x), \theta(y)], \theta(z)]+\varepsilon(z, x) \varepsilon(D, x)[[\theta(x), \delta(y)], \theta(z)] \\
& +\varepsilon(z, x) \varepsilon(D, x) \varepsilon(D, y)[[\theta(x), \theta(y)], D(z)] \\
& +\varepsilon(x, y)[[\delta(y), \theta(z)], \theta(x)]+\varepsilon(x, y) \varepsilon(D, y)[[\theta(y), \delta(z)], \theta(x)] \\
& +\varepsilon(x, y) \varepsilon(D, y) \varepsilon(D, z)[[\theta(y), \theta(z)], D(x)] \\
& +\varepsilon(y, z)[[\delta(z), \theta(x)], \theta(y)]+\varepsilon(y, z) \varepsilon(D, z)[[\theta(z), \delta(x)], \theta(y)] \\
& +\varepsilon(y, z) \varepsilon(D, z) \varepsilon(D, x)[[\theta(z), \theta(x)], D(y)] \\
= & \varepsilon(z, x)[[\delta(x), \theta(y)], \theta(z)]+\varepsilon(z, x) \varepsilon(D, x)[[\theta(x), \delta(y)], \theta(z)] \\
& +\varepsilon(z, x) \varepsilon(D, x) \varepsilon(D, y)[[\theta(x), \theta(y)], \delta(z)] \\
& +\varepsilon(x, y)[[\delta(y), \theta(z)], \theta(x)]+\varepsilon(x, y) \varepsilon(D, y)[[\theta(y), \delta(z)], \theta(x)] \\
& +\varepsilon(x, y) \varepsilon(D, y) \varepsilon(D, z)[[\theta(y), \theta(z)], \delta(x)]
\end{aligned}
$$




$$
\begin{aligned}
+ & \varepsilon(y, z)[[\delta(z), \theta(x)], \theta(y)]+\varepsilon(y, z) \varepsilon(D, z)[[\theta(z), \delta(x)], \theta(y)] \\
+ & \varepsilon(y, z) \varepsilon(D, x) \varepsilon(D, z)[[\theta(z), \theta(x)], \delta(y)] \\
= & \varepsilon(D, z)(\varepsilon(z, D) \varepsilon(z, x)[[\delta(x), \theta(y)], \theta(z)]+\varepsilon(x, y) \varepsilon(D, y)[[\theta(y), \theta(z)], \delta(x)] \\
& +\varepsilon(y, z)[[\theta(z), \delta(x)], \theta(y)]) \\
+ & \varepsilon(D, x)(\varepsilon(x, D) \varepsilon(x, y)[[\delta(y), \theta(z)], \theta(x)]+\varepsilon(y, z) \varepsilon(D, z)[[\theta(z), \theta(x)], \delta(y)] \\
& +\varepsilon(z, x)[[\theta(x), \delta(y)], \theta(z)]) \\
+ & \varepsilon(D, y)(\varepsilon(y, D) \varepsilon(y, z)[[\delta(z), \theta(x)], \theta(y)]+\varepsilon(z, x) \varepsilon(D, x)[[\theta(x), \theta(y)], \delta(z)] \\
& \quad+\varepsilon(x, y)[[\theta(y), \delta(z)], \theta(x)]) \\
= & 0 .
\end{aligned}
$$

Note that $\delta$ is a $\theta$-prederivation by Corollary 2.5. Therefore, $D$ is a generalized $\theta$-prederivation of $L$ with respect to a $\theta$-prederivation $\delta$ by Theorem 2.11

As before, one can prove the following theorems.

Theorem 2.13. $D$ is a generalized $(\theta, \varphi)_{2}$-prederivation of $L$ with respect to a $(\theta, \varphi)_{2}$-prederivation $\delta$ if and only if $D$ is a generalized Jordan $(\theta, \varphi)_{2}$-prederivation of $L$ with respect to a $(\theta, \varphi)_{2}$-prederivation $\delta$ such that

(i) $[[\delta(x), \theta(y)],(\varphi-\theta)(z)]=\varepsilon(\delta, x)[[\theta(x), \delta(y)],(\varphi-\theta)(z)]$,

(ii) $\varepsilon(z, x) B^{\prime}(x, y, z)+\varepsilon(x, y) B^{\prime}(y, z, x)+\varepsilon(y, z) B^{\prime}(z, x, y)=0$, where $x, y, z \in L$ and $B^{\prime}(x, y, z)=[[\delta(x), \theta(y)], \theta(z)]+\varepsilon(D, x)[[\theta(x), \delta(y)], \varphi(z)]+$ $\varepsilon(D, x) \varepsilon(D, y)[[\varphi(x), \varphi(y)], D(z)]$.

Theorem 2.14. $D$ is a generalized $(\theta, \varphi)_{3}$-prederivation of $L$ with respect to a $(\theta, \varphi)_{3}$-prederivation $\delta$ if and only if $D$ is a generalized Jordan $(\theta, \varphi)_{3}$-prederivation of $L$ with respect to a $(\theta, \varphi)_{3}$-prederivation $\delta$ such that

(i) $[[\delta(x),(\theta-\varphi)(y)], \theta(z)]=\varepsilon(\delta, x)[[(\theta-\varphi)(x), \delta(y)], \theta(z)]$,

(ii) $\varepsilon(z, x) B^{\prime \prime}(x, y, z)+\varepsilon(x, y) B^{\prime \prime}(y, z, x)+\varepsilon(y, z) B^{\prime \prime}(z, x, y)=0$,

where $x, y, z \in L$ and $B^{\prime \prime}(x, y, z)=[[\delta(x), \theta(y)], \theta(z)]+\varepsilon(D, x)[[\varphi(x), \delta(y)], \theta(z)]+$ $\varepsilon(D, x) \varepsilon(D, y)[[\varphi(x), \varphi(y)], D(z)]$.

Remark 2.15. Corollary 2.12 can also be concluded from Theorem 2.13 or Theorem 2.14 since for any $x, y, z \in L, B(x, y, z)=B^{\prime}(x, y, z)=B^{\prime \prime}(x, y, z)$ when $D$ is a generalized Jordan $\theta$-prederivation.

\section{ACKNOWLEDGEMENTS}

The authors would like to thank the referee for valuable comments and suggestions on this article.

\section{REFERENCES}

[1] Bajo, I. Lie algebras admitting non-singular prederivations. Indag. Math. (N.S.) 8 (1997), no. 4, 433-437. MR 1621901 
[2] Bergen, J.; Passman, D. Delta ideals of Lie color algebras, J. Algebra 177 (1995), no. 3, 740-754. MR 1358484

[3] Brešar, M. Jordan mappings of semiprime rings. J. Algebra 127 (1989), no. 1, 218-228. MR 1029414

[4] Burde, D. Affine cohomology classes for filiform Lie algebras. In: Crystallographic groups and their generalizations (Kortrijk, 1999), 159-170, Contemp. Math. 262, Amer. Math. Soc., Providence, RI, 2000. MR 1796131

[5] Burde, D. Lie algebra prederivations and strongly nilpotent Lie algebras. Comm. Algebra 30 (2002), no. 7, 3157-3175. MR 1914991

[6] Burde, D.; Moens, W. Periodic derivations and prederivations of Lie algebras. J. Algebra 357 (2012), 208-221. MR 2905250

[7] Chen, L.; Ma, Y.; Ni, L. Generalized derivations of Lie color algebras. Results Math. 63 (2013), no. 3-4, 923-936. MR 3057346.

[8] Feldvoss, J. Representations of Lie colour algebras, Adv. Math. 157 (2001), no. 2, 95-137. MR 1813428

[9] Herstein, I. Jordan derivations of prime rings. Proc. Amer. Math. Soc. 8 (1957), 1104-1110. MR 0095864

[10] Jacobson, N. A note on automorphisms and derivations of Lie algebras. Proc. Amer. Math. Soc. 6 (1995), 281-283. MR 0068532

[11] Ji, P.; Wang, L. Lie triple derivations of TUHF algebras. Linear Algebra Appl. 403 (2005), 399-408. MR 2140293

[12] Leger, G.; Luks, E. Generalized derivations of Lie algebras. J. Algebra 228 (2000), no. 1, 165-203. MR 1760961

[13] Ma, Y.; Chen, L. On generalized Jordan prederivations and generalized prederivations of Lie superalgebras. Adv. Math. Phys. 2014, Art. ID 401238, 9 pp. MR 3259176

[14] Miers, C. Lie triple derivations of von Neumann algebras. Proc. Amer. Math. Soc. 71 (1978), no. 1, 57-61. MR 0487480

[15] Moens, W. A characterisation of nilpotent Lie algebras by invertible Leibniz-derivations. Comm. Algebra 41 (2013), no. 7, 2427-2440. MR 3169402

[16] Montgomery, S. Constructing simple Lie superalgebras from associative graded algebras. J. Algebra. 195 (1997), no. 2, 558-579. MR 1469640.

[17] Müller, D. Isometries of bi-invariant pseudo-Riemannian metrics on Lie groups. Geom. Dedicata 29 (1989), no. 1, 65-96. MR 0989188.

[18] Najati, A. Generalized derivations on Lie triple systems. Results Math. 54 (2009), no. 1-2, 143-147. MR 2529633

[19] Najati, A. On generalized Jordan derivations of Lie triple systems. Czechoslovak Math. J. 60(135) (2010), no. 2, 541-547. MR 2657968

[20] Rittenberg, V.; Wyler, D. Generalized superalgebras. Nuclear Phys. B 139 (1978), no. 3, 189-202. MR 0491859

[21] Scheunert, M. Generalized Lie algebras. J. Math. Phys. 20 (1979), no. 4, 712-720. MR 0529734

[22] Su, Y.; Zhao, K.; Zhu, L. Simple Lie color algebras of Weyl type, Israel. J. Math. 137 (2003), 109-123. MR 2013351

[23] Wang, H.; Li, Q. Lie triple derivation of the Lie algebra of strictly upper triangular matrix over a commutative ring. Linear Algebra Appl. 430 (2009), no. 1, 66-77. MR 2460499 
[24] Wilson, M. Delta methods in enveloping algebras of Lie color algebras, J. Algebra 175 (1995), no. 2, 661-696. MR 1339662

[25] Zhang, J.; Wu, B.; Cao, H. Lie triple derivations of nest algebras. Linear Algebra Appl. 416 (2006), no. 2-3, 559-567. MR 2242444

Chenrui Yao

School of Mathematics and Statistics, Northeast Normal University, Changchun 130024, China

Yao $M a$

School of Mathematics and Statistics, Northeast Normal University, Changchun 130024, China

Liangyun Chen ${ }^{凶}$

School of Mathematics and Statistics, Northeast Normal University, Changchun 130024, China

chenly640@nenu.edu.cn

Received: October 15, 2017

Accepted: June 26, 2018 